\title{
La gestion publique des émotions et les ruses de l'intelligence
}

\author{
Julien Thiburce ${ }^{1, *}$ \\ ${ }^{1}$ CNRS, ICAR (UMR 5191), ENS de Lyon, 69007 Lyon, France
}

\begin{abstract}
Résumé. Dans un dialogue entre la sémiotique, l'analyse conversationnelle et l'analyse du discours, la notion de ruse affirmera sa force heuristique pour penser les articulations feintes et déjouées entre ethos (valeurs et implication éthique), logos (rationalité et implication raisonnée) et pathos (sensibilité et implication affective). En lien avec l'analyse d'un extrait d'enregistrements de balades urbaines guidées réalisés dans le cadre de notre thèse, nous proposerons une réflexion sur le caractère approprié d'une conduite en groupe, selon le point de vue des participants. À partir des dynamiques transitives et réflexives de la gestion des émotions en public, nous réfléchirons aux enjeux socioaffectifs de formes énonciatives comme l'ironie. Dans une perspective éthnométhodologique, nous porterons un regard sur les effets perlocutoires des échanges entre les participants de manière transversale à la séquentialité de l'interaction, à la progression thématique d'un discours en groupe et à la gestion collective des ethos.
\end{abstract}

\begin{abstract}
In a dialogue between semiotics, conversational analysis and discourse analysis, the notion of trick will assert its heuristic strength to think about the fake and thwarted articulations between ethos (values and ethical involvement), $\log o s$ (rationality and reasoned involvement) and pathos (sensitivity and affective involvement). In relation to the analysis of an excerpt from recordings of guided urban walks made in our doctoral research, we will propose a reflection on the appropriateness of a conduct in public, from the participants' point of view. From the dynamics of the management of emotions in public, we will reflect on the socio-affective stakes involved in enunciative forms such as irony. In an ethnomethodological perspective, we will look at the the perlocutionary effects of group speech across the sequentiality of interaction, the thematic progression of discourse and the collective management of ethos.
\end{abstract}

\section{Introduction à la sémiotisation des émotions en interaction}

Dans le cadre de notre recherche doctorale, nous avons mené une étude empirique des formes d'appropriation narrative et affective de la ville à partir d'interactions en situation de balades urbaines guidées programmées par les musées Gadagne (musée d'histoire de la Ville de Lyon), sur le thème de l'Esprit skate. Cette balade est alors l'occasion d'un débat

\footnotetext{
*julien.thiburce@ens-lyon.fr
} 
entre des habitants sur des manières de concevoir la ville à travers le prisme des pratiques du skateboard sur le plan philosophique (l'esprit en tant que spirit) et sur le plan d'une expérience incarnée d'environnements urbains (esprit en tant que mind). Au cours des échanges entre les guides et les participants, de manière programmée ou ajustée, l'expérience in vivo de la balade se trouve en prise avec d'autres expériences quotidiennes (hors-balade urbaine) sur les plans de perception, des savoirs, des rôles sociaux et des affects. Ce qui est perçu, énoncé et thématisé dans le parcours en groupe entre en résonnance avec les autres expériences déjà vécues et celles à vivre, que ce soit selon une anticipation des étapes de l'itinéraire ou selon un mouvement de projection utopique (une ville dans laquelle chacun se reconnaitrait, enfin). En interaction constante, ces expériences interrogent le sens de ce qui se déroule dans les échanges en groupe, sur le fond d'un récit social dont plusieurs versions chez les institutions, les guides et les visiteurs peuvent entrer en concurrence ou en congruence (en prenant des extrêmes à gros traits, "la pratique du skateboard est irrespectueuse de la ville et de ses habitants et mérite d'être pénalisée", "la pratique du skate, considérée en tant que pratique sportive et artistique, sublime les relations entre les agents sociaux et leur territoire et mérite d'être prise en compte de manière positive dans les projets urbains").

À partir d'un extrait de l'enregistrement audiovisuel de ces balades urbaines, cet article vise à mettre en visibilité en quoi les interactions dans le cours de ces balades urbaines guidées donnent matière à réflexion sur les processus de sémiose des émotions (couplage des plans de l'expression et du contenu), sur les modalités linguistiques et sémiotiques par lesquelles les participants négocient leurs identités sociales à travers une prise de parole en public. En appréhendant cette sémiose des émotions, d'une part, au niveau des contenus référentiels élaborés par des interactants, qui décrivent des «états de chose » (ici, l'interaction est un «texte» à coproduire) et, d'autre part, au niveau des contenus relationnels, où des énoncés en interaction « contribuent à instituer entre les interactants un lien socio-affectif particulier» [1, p. 9], notre intention est en effet de décrire en quoi les prises de paroles (i) exhibent le caractère instable du travail de figuration [2], (ii) questionnent les passages entre passions individuelles et passions collectives et (iii) interrogent la négociation du terrain de pratiques sémiotiques toujours en devenir.

Ainsi, on étudiera les tensions sous-jacentes à un jeu de la part des participants sur leurs propres identités actorielles (les rôles incarnés in vivo et les valorisations afférentes), à partir d'une observation des formes sémiotiques et linguistiques par lesquelles des agents sociaux négocient leurs perceptions et leurs affects en interaction. C'est ici même que les ruses de l'intelligence théorisées par Marcel Détienne et Jean-Pierre Vernant [3] selon une lecture critique de textes grecs anciens nous parait pertinente pour l'étude des modalités énonciatives par lesquelles se déploie un double regard sur des états émotionnels (transitif et réflexif). Selon leurs mots, "multiple et polymorphe, [l'intelligence de la ruse] s'appliquait à des réalités mouvantes qui ne se prêtent ni à la mesure précise ni au raisonnement rigoureux ». Cette focalisation sur la notion de ruses permettra ainsi de travailler les enjeux socio-pragmatiques d'une maîtrise de soi et de l'entour de l'interaction, en distinguant une ruse passive, à savoir un ephemeros dont les métamorphoses «sont produites par la peur », et une ruse active, à savoir un polutropos dont les métamorphoses " sont dues à l'astuce », un " homme aux mille tours, une mètis dont la souplesse ne paraît se plier aux circonstances que pour les dominer plus sûrement » [3, p. 48].

En lien avec notre analyse d'un cas spécifique, nous proposerons aussi des réflexions théoriques sur la place des formes sémiotiques dans les liens entre émotions et actions, réflexions qui n'auront pas pour autant la prétention de pouvoir s'appliquer à une diversité et une hétérogénéité majeure d'autres situations. Dans le couplage entre des outils de la sémiotique qui s'intéresse aux médiations, de l'analyse conversationnelle qui s'intéresse au caractère séquentiel des interactions, en partant du point de vue des participants, et de l'analyse du discours qui vise à décrire la construction des points de vue, des 
responsabilités et des engagements en énoncé, on s'intéressera particulièrement aux formes d'anticipation et de rétroaction des émotions qui agissent sur l'énonciation des participants.

\section{1 Émotions, affects et passions : incarner une position "face ouverte" ou "face cachée"}

Dans la perspective d'une enquête sémiotique des pratiques en interaction, les affects (perçus) tiennent une place charnière entre émotions (vécues) et passions (discursivisées) [4] dans la manière dont les participants gèrent leurs faces positives et négatives en interaction. Par notre étude menée à partir d'une pratique in vivo, on cherche notamment à problématiser en quoi, dans leurs participations "face ouverte" et "face cachée" à une interaction, des individus et des collectifs instaurent, énoncent et implémentent des pratiques en public, en étant confrontés au caractère fuyant de leur propre expérience de la ville en groupe. Une telle perspective permet de décrire les processus selon lesquels le fait de jouer et de déjouer des conflits affectifs en interaction est directement lié à la gestion des conflits narratifs en situation, à la gestion d'une (dis)continuité entre des positionnements actoriels conçus depuis le point de vue d'un individu et ceux qui émergent depuis le point de vue de ses coparticipants. En ligne de fond, on travaille sur les processus de gestion de l'attention [5; p. 273],[6] selon lesquels les interactants conçoivent leurs prises de parole, au sens d'un design de l'interaction, en éclairant leur portée tantôt clivante, tantôt collectivisante (tel qu'on pourra le voir en section 2.3). En effet, notre réflexion sémiotique sur la gestion des affects à partir d'un discours en interaction, dans la ville et sur la ville, demande de s'attarder sur les relations matérielles, affectives et symboliques entretenues aux espaces repérés et balisés en amont de la visite par les guides (pour concevoir l'itinéraire), mais aussi aux espaces vécus et traversés par chacun et chacune des participants, dans leur vie respective.

Dans l'observation des pratiques sociales et langagière in situ, il y a une relation d'intrication à étudier entre, d'une part, des modes de présence à l'environnement en train d'émerger ici et maintenant par une expérience in vivo et, d'autre part, des formes langagières dont les interactants supposent ou affirment qu'elles sont stabilisées et partagées. Ainsi, notre proposition n'est pas tant d'élaborer une typologie des émotions ou de discuter les grilles descriptives d'analyse du discours sur la sémiotisation des émotions, comme a pu le faire finement Raphael Micheli [7] en distinguant les émotions dites, montrées et étayées ${ }^{\dagger}$. Nous tendons plutôt à repérer les relations qui s'instaurent entre les participants dans la gestion dynamique et collaborative d'une sémiotisation d'un état émotif, que cet état ait été réellement éprouvé ou bien joué et feint en discours. Par ailleurs, un mot mérite d'être dit ici sur le métalangage mobilisé pour référé à l'interprétation et l'énonciation des états émotifs. Dans le sillage de Raphael Micheli, l'une des motivations épistémologiques à mobiliser le terme "sémiotisation » est qu'il traduit la complexité interne à l'élaboration d'une interprétation des émotions, selon des processus d'auto-

\footnotetext{
${ }^{\dagger}$ Raphaël Micheli [7, p. 35], distingue l'émotion dite en partant « de l'idée que le dire - en tant que mode de sémiotisation - implique la présence, dans l'énoncé, d'un " terme d'émotion ». Il propose ensuite une " tentative de définition » de l'émotion montrée comme celle pour laquelle "l'allocutaire est conduit à inférer que le locuteur - ou, en cas de disjonction énonciative, l'énonciateur - éprouve une émotion, sur la base d'une relation de cooccurrence supposée entre, d'une part, l'énonciation d'un énoncé présentant ces caractéristiques et, d'autre part, le fait d'éprouver une émotion » [7, p. 64]. Enfin, dans le cas de l'émotion étayée, "l'allocutaire part d'une situation discursivement schématisée, et en infère une certaine émotion qu'il considère comme son effet légitime, en vertu de normes socio-culturelles qui apparient des types d'évaluation des situations, d'une part, et des types d'émotion, d'autre part » [7, p. 120].
} 
attribution et d'allo-attribution impliqués dans la gestion des points de vue sur le théâtre des énonciations.

\subsection{L'appropriation : une notion clé pour l'étude de la sémiose des émotions}

Aussi, il nous faut expliciter en quoi l'appropriation a selon nous une valeur heuristique pour l'étude de la sémiose des émotions en interaction. Entre le fait de "faire siennes" des pratiques langagières et le fait de "se rendre propre" [8 ], [9] à une situation d'interaction, l'appropriation s'affirme comme notion clé pour problématiser une articulation entre, d'une part, les phases d'une pratique et d'une expérience en cours, où chaque personne fait face à l'immédiat et aux contingences environnementales, et d'autre part, le feuilletage des modes d'existence individuels et collectifs aux espaces publics, qui sont soumis à la conciliation d'une diversité de normes et de cadres pragmatiques.

En parcours, un sens des pratiques sociales dans la ville émerge par des interactions entre des acteurs sociaux qui construisent et ajustent leurs perceptions, leurs savoirs et leurs affects, en cherchant à stabiliser l'horizon de leurs expériences vers lequel s'orienter. Comme pour toute interaction, il y a une indétermination constitutive (i) vis-à-vis de ce qui va faire l'objet de leur discours et (ii) quant aux interprétations et valorisations de leurs conduites respectives. L'un des enjeux de l'étude de la sémiotisation des émotions en interaction est de caractériser en quoi la gestion dynamique des prises de parole met en tension la production d'un discours et d'un récit sur la ville en groupe, entre au moins deux positions : d'une part, la position de réception d'une parole à interpréter et à s'approprier ("mon interprétation de ce qui est dit est-elle juste ?"; "en quoi et jusqu'où mon point de vue sur telle question est-il semblable ou différent de celui des autres ?") ; d'autre part, la proposition d'une parole dont la pertinence demande d'être évaluée par rapport à ce qui a été énoncé et sera énoncé par chacun ("de quoi parle-t-on et ma prise de parole est-elle cohérente par rapport à la trame du discours en train de se tisser?"; "comment rendre compte de la diversité des expériences et des points de vue sur les pratiques des espaces publics?").

En procédant à une analyse d'interactions à partir d'enregistrements audiovisuels plutôt qu'à une étude de la sémiose des émotions à travers des textes déjà produits (méthode majoritaire en analyse du discours et en sémiotique), nous rendrons compte de cette dynamicité qui caractérise les ajustements entre un point de vue "sien" et un point de vue "autre", en amont et en aval de chaque prise de parole. La focalisation sur l'appropriation en tant que processus consistant à «faire sien » un environnement et à "(se) rendre propre (à) » un environnement matériel, affectif et symbolique, nous permettra de montrer en quoi l'énonciation se révèle être le terrain d'un conflit relatif au fait même de prendre la parole selon des cadres pragmatiques (re)définis en situation. Nous pourrons ainsi observer les ruses énonciatives mises en œuvre par les interactants pour négocier le caractère potentiellement inapproprié de leur prise de parole, selon leur perspective ("peut-on couper la parole à un interlocuteur sans problème ?"; "en prenant la parole, empêche-t-on quelqu'un de parler?"; "se verrait-on attribuer le rôle de participant irrespectueux des autres?").

\footnotetext{
* «Un modèle d'analyse adéquat doit pouvoir saisir le « langage émotionnel » en tant qu'il engage non seulement des processus d'auto-attribution, mais aussi d'allo-attribution, lors desquels un locuteur attribue une émotion à d'autres que lui-même. À ce titre, si l'on dit qu'un locuteur " sémiotise » une émotion, on laisse ouverte la question de savoir à qui cette émotion est attribuée par l'énoncé. » (Micheli, 2014 : 19) [7]
} 


\section{2 Étude sémiotique d'un cas spécifique}

\subsection{Quels positionnements séquentiel, thématique et éthique appropriés ?}

Dans cet horizon épistémologique, nous allons étudier ces dynamiques à partir d'un extrait d'une balade qui s'est déroulée le 31 mai 2016. Au deuxième point d'arrêt du parcours, les participants échangent sur les modalités de pratiques des photographes et vidéastes dans le milieu du skateboard. Alors que les guides clôturent le thème de leur discours en initiant un départ vers le point d'arrêt suivant, une participante tente de revenir sur une question déjà traitée dans les échanges précédents. Nous verrons qu'elle s'impose dans l'espace interactionnel pour faire entendre sa voix sur un ton de manière (dé)joué.

Sans trop en dire sur cet extrait avant de le retranscrire ci-après, cette prise de parole s'avère heuristique quant à la sémiotisation des émotions opérée par les participants sur le plan :

- de l'organisation séquentielle de la parole-en-interaction (comment/quand prendre la parole et faire en sorte que les coparticipants écoutent, sans les brusquer ?),

- de la progression thématique du discours en interaction (comment/quand changer de thème sans paraître hors-sujet, comme si l'on n'avait pas prêté attention à la parole des autres ?),

- de la cogestion des ethos (comment impulser une trajectoire d'action au groupe sans paraître dictateur ni irrévérencieux ?).

Ainsi, nous nous intéressons tout particulièrement à l'organisation séquentielle des prises de parole pour ce qu'elle engage en termes de cohérences et cohésions discursives, sur le plan de la progression thématique, et en termes de la préservation des faces [2], sur le plan de l'ethos. En abordant l'extrait vidéo avec un regard transversal à ces trois plans, nous pourrons ainsi étudier sur pièces comment et quand des ruses énonciatives sont mises en œuvre dans la co-construction d'une image de soi vis-à-vis des interactants (des jeux entre personne et personnage), mais également dans le déploiement d'une relation socioaffective avec les participants à une activité qui est avant tout groupale (la mise en partage de perceptions, de savoirs et d'affects fait appel à une intelligence collective, une confiance interpersonnelle). Dans notre approche, le caractère approprié des positionnements séquentiel, thématique et éthique n'est bien évidemment pas abordé selon une perspective prescriptive et normative. Ce qui nous importe est de penser la transformation des normes en groupe et ses effets sur la scène de l'interaction en termes de conflits entre des cadres sociaux [10], en l'occurrence les règles qui agissent au cours de la balade urbaine et celles hors balade urbaine, selon une perspective ethnométhodologique [11]. Quant à la gestion de l'attention mentionnée plus haut, il sera notamment intéressant d'observer la place que tiennent les jeux de regards, les gestes de pointage et les sourires entre les participants, dans la régulation collective de l'interaction.

\subsection{La prise des paroles entre usage introjecté et ruse opportune}

Dans cet extrait, on trouve une dynamique intéressante sur le plan interactionnel, du point de vue de la négociation des formes langagières, comme sur le plan énonciatif, du point de vue de la prise en compte et de la prise en charge de points de vue autres que le sien propre. Nous transcrivons d'abord cet extrait ${ }^{\S}$ de manière à avoir un regard sur le développement

\footnotetext{
$\S$ Nous avons appliqué les conventions constituées par le groupe ICOR en analyse conversationnelle : http://icar.univ-lyon2.fr/projets/corinte/documents/2013_Conv_ICOR_250313.pdf.

Les noms des participants sont anonymisés: GU1 et GU2 signifient GUide 1 et GUide 2 ; VF1 signifie Visiteur Femme 1. Aussi, nous réalisons des captures d'écran de l'extrait vidéo étudié, dont la position est marquée dans la transcription : le symbole \#1 positionne l'image 1 dans l'interaction.
} 
progressif de l'échange au niveau micro-discursif, puis nous en proposerons une synthèse pour en saisir les enjeux macro-discursifs, à la fois sur le plan du genre de pratique de médiation de la «balade urbaine » et sur le plan du discours du parcours dans son entier.

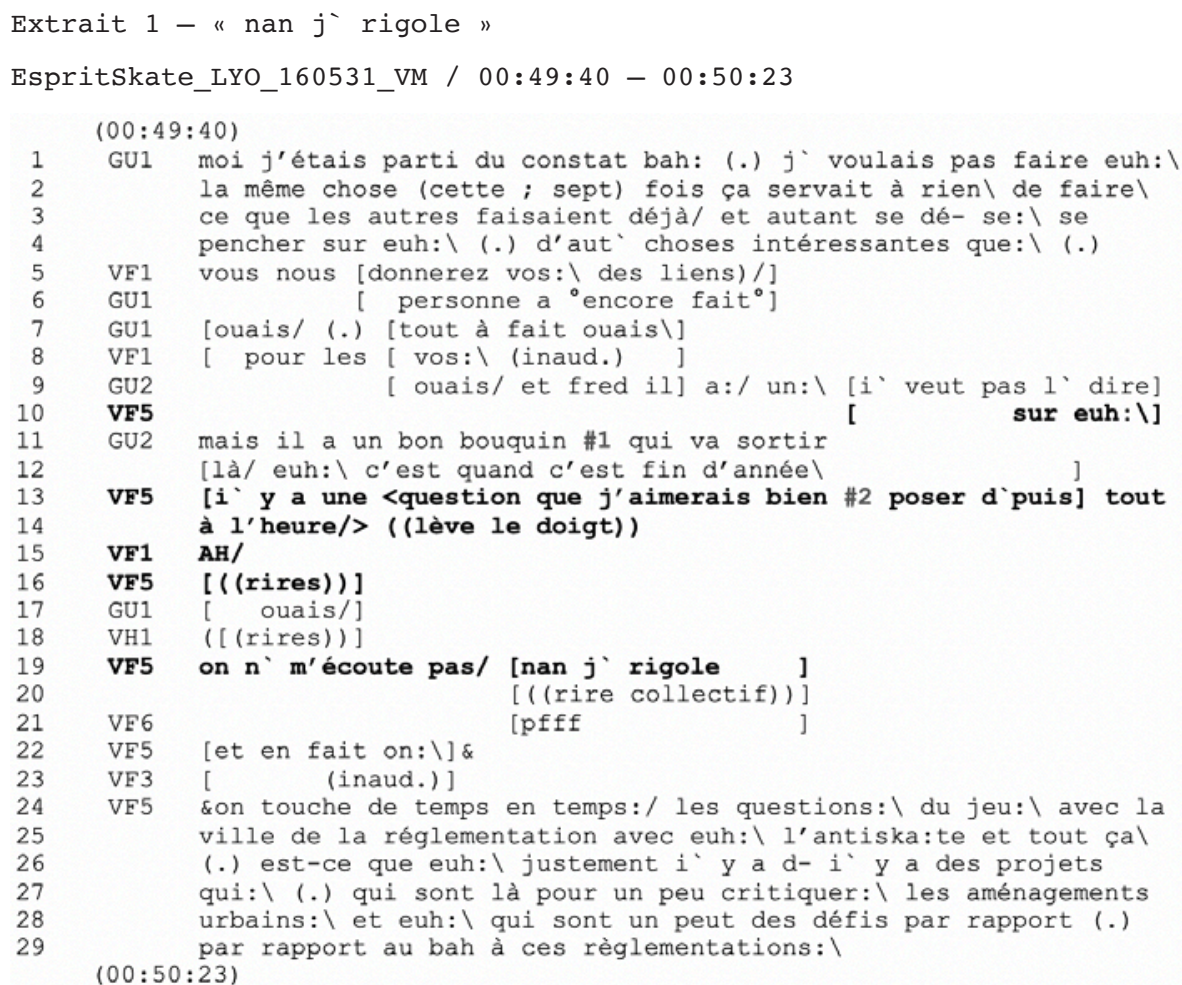

Dans le cours de cet extrait, GU1 dépeint son rôle de photographe et vidéaste en disant qu'il se penche sur «d'autres choses intéressantes que [...] personne a encore fait » (lignes 1-4 et 6). VF1 prend alors la parole de manière spontanée et auto-initiée, en chevauchement à GU1, en posant la question de savoir si les guides ont des espaces où publier leurs photos et leurs vidéos (ligne 5).

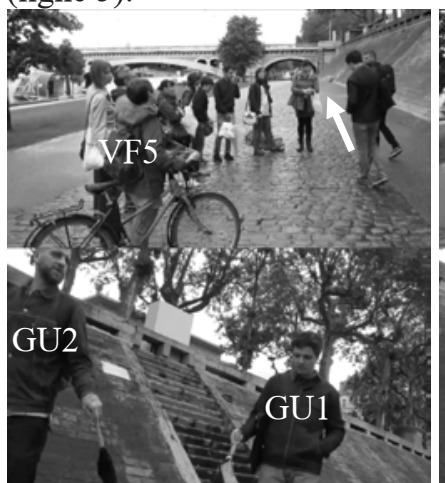

$\# 1$

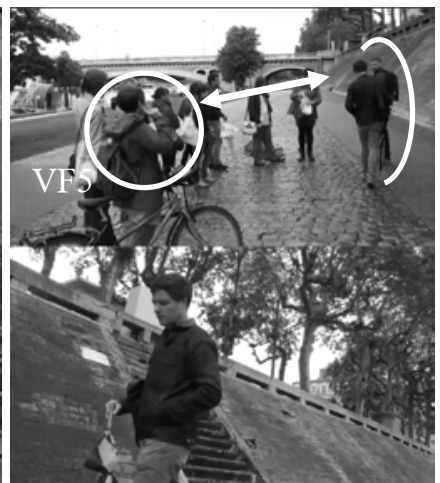

\#2

Fig. 1 Déplacement initié par GU2 (\#1, à gauche) et focalisation attentionnelle (\#2, à droite)

Ce à quoi GU2 répond que «Fred a un bon bouquin » qui sortira à la « fin d'année » 2016 (lignes 9 et 11-12), tout en initiant un départ vers le troisième point d'arrêt dénommé Small Place par les guides et les skateurs (Figure 1, \#1, flèche blanche). C'est un moment critique dans le déroulement de la balade, en ce qu'il marque une transition entre une 
séquence de médiation (au spot de skateboard où se trouve le groupe) et une séquence de déplacement (vers le point d'arrêt suivant). VF5 s'impose en interrompant la trajectoire que GU2 initie plus par son corps que par son discours verbal : elle a « une question » qu'elle « aimerait bien poser depuis tout à l'heure » (lignes 13-14).

Dans toute la multimodalité de l'échange, elle occupe l'espace interactionnel par sa voix et par son corps en levant le doigt presque scolairement (Figure 1, \#2, cercle blanc), en vue de retenir l'attention des guides et d'obtenir une réponse de leur part quant à un axe thématique qu'elle souhaite approfondir: la place de la réglementation dans les projets urbains, une question épineuse, soumise à controverse et génératrice de tensions dans les échanges au cours de la balade urbaine et hors balade urbaine. Ceux-ci interrompent alors leur marche en direction de la Small Place, reviennent sur leurs pas et reprennent la position adoptée précédemment.

\subsection{Le design progressif des contours de l'interaction}

Dans la séquentialité de l'interaction, des pre-beginning tels que la gesticulation de VF5, son mouvement en avant vers les guides et sa prise de parole («sur euh:\», ligne 10) amorcent cette question. Étudiés notamment par Lorenza Mondada [12] pour leur rôle dans l'articulation des prises de parole de chacun des participants, entre incipient (vis-à-vis de la gestion des points de transitions pertinents, notamment) et recipient (négociation de l'adresse et co-construction des énoncés sur les plans de l'expression et du contenu), ces pre-beginnings représentent ici une phase majeure de la gestion de la transitivité de cet échange. Vis-à-vis des formes de participation à l'interaction, ils permettent de gérer sa présence dans un espace en tâtonnant dans sa prise de parole, en ne s'imposant pas de manière abrupte et en cherchant à entrer en relation avec les autres, de manière dynamique (on prend en compte les actions déjà réalisées et celles potentialisées par l'action en cours) et bidirectionnelle (on gère les relations sociales et symboliques, pour lesquelles chacun prend part à la (re)définition de ce qui est approprié et pertinent par son positionnement). Il est à noter que la dimension temporelle de l'action initiée par VF5, sur le fond des paroles déjà déployées par les autres participants, tient une place importante dans le design de son énoncé : en disant «il y a une question que j'aimerais poser depuis tout à l'heure », (i) elle dénote la tension temporelle qui s'instaure entre le temps de parole qu'elle va prendre et celui déjà pris par d'autres et (ii) elle intensifie le demande d'attention symbolisée par la main levée scolairement (elle se retient depuis suffisamment longtemps pour que les coparticipants lui accordent de l'attention).

Cette introduction provoque alors la réaction verbale et/ou gestuelle des coparticipants, notamment de VF1 qui s'exclame par un «ah» (ligne 15). On interprète celui-ci comme relevant moins d'un changement d'état (épistémique ou affectif, par exemple), d'un change of state token selon la terminologie mobilisée entre autres par John Heritage [13], que de l'expression spontanée d'une expectation et d'une invitation à en dire plus. VF5 poursuit sa prise de parole en instillant un décalage de ton ${ }^{* *}$ par rapport à son introduction, sur la

\footnotetext{
** Dans une approche systématisante, la distinction opérée par Alfredo Lescano [15, p. 48-52], reprise par Julien Longhi [16] distingue : (i) « le ton de locuteur » qui est identifié au "locuteur en tant que tel" de Ducrot [17], à savoir l'image que l'énoncé construit de son auteur »; (ii) le «ton de Monde », qui est "proche de ce que Benveniste [18] appelait l'histoire, ces discours censés ne pas avoir de locuteur, et aussi de ce que Berrendonner [19] nommait le Fantôme de la vérité »; (iii) le «ton de Témoin » qui « entremêle » les « savoirs » et « perceptions » du locuteur et construit en même temps l'image d'un interlocuteur « qui n'a pas accès à ce dont on parle ».

Aussi, l'expression "de bon ton" permet de mettre en lumière les forces qui agissent sur l'interaction dans l'ajustement de la tonalité des (dés)accords entre des agents sociaux. En effet, si l'on dit d'un mot ou d'un geste qu'ils sont "de bon ton", c'est non seulement parce qu'une action se déploie en
} 
composante dialogique de son énonciation [14]. Avant de poser sa question, elle déjoue son imposition dans l'espace interactionnel en jouant la plainte, la mise à l'écart et en feintant la culpabilisation des autres : « on ne m'écoute pas nan je rigole» (ligne 19). Cette prise de parole nous questionne quant aux modes de présence à l'espace interactionnel, en ce qu'elle semble déjouer un éventuel incident diplomatique.

En gérant le caractère approprié de sa conduite d'action dans un collectif où agissent des forces contraires (les autres veulent aller là-bas, alors qu'elle souhaite rester ici), elle recourt à un jeu sur sa propre identité actorielle. En disant «on ne m'écoute pas », ne chercherait-elle pas à atténuer un embarras qui serait potentiellement causé par sa propre intervention, contraignant le groupe à dévier sa trajectoire, en faisant jouant elle-même la plante ? Et même si ce jeu linguistique, social et symbolique pourrait déjà être perçu par la simple rupture de ton et de registre qu'elle instille dans sa voix, trop "franche" pour être "vraie", elle fait comprendre de manière explicite aux autres participants que son intervention relève de ce que nous pourrions appeler un jeu d'adresse en cultivant la polyphonie de cette expression (discursive et comme habileté). En effet, dans le déploiement séquentiel de son énonciation, la formule «non je rigole » tient lieu de seuil discursif où elle dit et montre $e^{\dagger \dagger}$ aux coparticipants (i) qu'elle joue sur les rôles en train d'être tenus par elle et par les autres et (ii) qu'elle a bien certaine maîtrise des effets pragmatiques des conduites sociales qui la lie aux autres.

\subsection{La coagulation appropriée d'affects antagoniques : vivre dans l'ironie}

Dans la gestion du caractère propre ou impropre de cette prise de parole, un dialogisme est ainsi à l'œuvre à travers une énonciation qui progresse sur la crête de la mimèsis et de la sémiosis d'une polémique. Précisément, c'est une forme d'autodialogisation [22, p. 84] qui, par anticipation, prévient les effets perlocutoires de sa prise de parole sur la scène de l'énonciation : au lieu de taire le conflit, la participante l'exacerbe, le rend présent en le négociant à la fois en négatif (elle le déjoue par ruse plutôt que de laisser poindre "pour de vrai") et en sens contraire (elle tient les autres pour responsables plutôt que d'être tenue elle-même pour responsable). L'ironie de la situation favorise ainsi la transformation des contenus référentiels et le passage d'une parole à la recherche de prises relationnelles.

En effet, la mobilisation de la forme impersonnelle par VF5 pour référer aux membres du groupe («on ne m'écoute pas ») atténue la valence négative des liens sociaux qui aurait pu être exprimée en plein et de manière frontale, par exemple à travers la mobilisation du pronom « vous» («vous ne m'écoutez pas »). La neutralisation d'une polarisation entre un «vous" et un «je » réalisée ici participe de cette tension entre l'instauration d'un geste énonciatif qui aurait pu être culpabilisant et d'un jeu sur les positions en train d'être revendiquées par soi, pour soi, et celles imputées à autrui. Cela débouche sur la conciliation

accord aux attentes relatives à un cadre social et pragmatique, mais c'est également parce qu'une action fluide, sans accrocs et à propos, rétablit une situation qui prenait un mauvais tour.

†† Nous faisons ici référence au couple notionnel dire et montrer développé par Wittgenstein, mobilisé en sciences du langage notamment par Maingueneau [20] à la suite de Ducrot [17] :

«Pour ma part, c'est en analyste du discours que j'aborde l'ethos, par conséquent en termes d'ethos discursif. Celui-ci implique à la fois l'ethos dit (ce que le locuteur dit sur lui-même, par exemple qu'il est un homme simple), et l'ethos montré (ce que montre sa manière d'énoncer). Cette distinction est exprimée chez Ducrot [17, p. 201] à travers l'opposition entre locuteur-L (le locuteur en tant qu'il est en train d'énoncer) et locuteur- $\lambda$ (le locuteur en tant qu'être du monde, hors de l'énonciation). L'ethos discursif se montre dans l'acte d'énonciation, il ne se dit pas dans l'énoncé ; il est perçu, mais il ne fait pas l'objet du discours. » [20]

Maingueneau rappelle que les analystes du discours distinguent cet ethos discursif d'un ethos prédiscursif, dans le sens que lui a donné Ruth Amossy [21, p. 70], en spécifiant sa dimension pragmatique, relative à des valorisations incorporées avant l'acte d'énonciation. 
ironique d'un énoncé de l'énonciateur E1 qui se positionne en faux sur ce qui est en train d'être dit et montré par l'énonciateur E2, position de dissensus interne à son propre discours bien perçue comme telle par les co-énonciateurs, au regard du rire collectif afférent.

Pour penser ces jeux d'anticipation et de rétroaction dans la mise en scène des voix et des corps dans l'espace interactionnels sur le plan sémiotique, il nous semble particulièrement éclairant de lier cette observation sur les ajustements de points de vue discursifs en interaction avec l'approche déployée en sémiotique par Pierluigi Basso Fossali [23] dont nous nous inspirons. Dans le cas spécifique que nous analysons, la sémiotisation des émotions en situation peut notamment être appréhendée à l'aune de la dialectique entre coagulation des affects et fluidification des interactions $\$$. En effet, l'ironie qui sous-tend ce jeu d'adresse mis en œuvre par VF5 procède à la fois d'une coagulation d'affects antagoniques (jouer à être blessé et à prendre suffisamment de distance pour en rire) et d'une fluidification d'une relation potentiellement polémique (jouer à être coupable d'accuser les autres). L'ironie manifestée entre le rôle perçu et le rôle énoncé, le rôle qu'elle se sent incarner et le rôle qu'elle donne à voir en public, permet alors de travailler cette dialectique entre coagulation et fluidification des affects (sur le plan théorique) à des jeux d'anticipation et de rétroaction dans la mise en scène des voix et des corps dans l'espace interactionnel (dans le cadre d'une analyse de corpus).

L'analyse de cet extrait, si banal et ordinaire qu'il puisse paraître au premier abord, nous invite alors à penser le couplage et le développement des outils des sciences du langage pour travailler "sur pièces" aux passages continus entre perception et énonciation en interaction. En reprenant notamment la systématisation proposée en 2.1 sur les positionnements séquentiel, thématique et éthique appropriés (selon le point de vue des participants), l'étude d'autres cas à un niveau de granularité fin permettrait de caractériser plus à fond les passages entre :

(i) l'irruption d'émotions ressenties en soi exprimée de manière spontanée, sans filtre, et le déploiement d'un regard réflexif exprimé sur le mode de la rétention, sur la plan de l'organisation séquentielle ;

(ii) les perceptions, interprétations et énonciation en privé et leur traduction publique en affects et en passions, sur le plan thématique d'une parole constituée en groupe ;

(iii) la manifestation locale de vécus de signification produisant des écarts et des connivences et le tissage d'une trame discursive d'un récit sur et dans lequel on n'est loin d'être le.a seul.e à pouvoir agir, sur le plan de la gestion collective des ethos.

\footnotetext{
\$ Pierluigi Basso Fossali précise alors que cette dialectique permet de préciser les relations entre perception et énonciation, sans accorder à l'une ou à l'autre un rôle privilégié dans la saisie du sens : "L'énonciation cherche par exemple à mobiliser le sens lorsque la perception semble avoir assigné des rôles actantiels stabilisés, voire figés. La prise d'initiative discursive permet donc de promouvoir une fluidification des relations en acte et d'envisager, à travers sa configuration fictive, une coagulation différente des rôles dans l'espace énoncé et énonciatif. Néanmoins, la coagulation discursive est à son tour toujours précarisée par la perception qui peut exhiber son caractère insubordonné et refluidifier les relations à travers la perméabilisation à l'environnement » [23, p. 66].
} 


\section{Pour une approche située des liens entre paroles, émotions et actions}

\subsection{Le rire et l'ironie comme antichambre interprétative}

Du point de vue de l'organisation séquentielle, en prenant du recul sur les situations déjà étudiées dans le corpus de la thèse, le rire et l'ironie s'affirment, parmi d'autres modalités sémiotiques, comme le terrain d'une gestion de la coprésence de positions contraires et le milieu de cohabitation d'affects hétérogènes. En effet, on ruse d'ironie et l'on se met à rire non seulement pour détendre l'atmosphère, mais aussi pour réduire l'atmosphérisation [24, p. 14] des positions déjà tenues ou celles que l'on voudrait tenir vis-à-vis de ses interlocuteurs. Le rire et l'ironie ne nous paraissent pas être seulement les indices d'un passage d'un rôle à un autre ou d'un état à un autre sur le plan idiosyncrasique : ils sont aussi une action à expérimenter de manière collective et concertée. Un espace des possibles se cultive alors dans les interstices entre une hétérogénéité de positions que l'on pourrait soi-même tenir au regard d'une situation spécifique.

Une piste de travail s'ouvre sur la recherche des modalités énonciatives à travers lesquelles les interactants opèrent une socialisation des "crises diplomatiques" qui les départagent et des conflits intrasubjectifs qu'ils éprouvent. Il s'agit alors de caractériser comment se négocie la marge d'un soi approprié sur le plan narratif et affectif entre le personnage énacté (un soi que l'on donne à voir à ses interlocuteurs) et les émotions éprouvées à un moment donné (un moi que l'on se sent incarner). En rigolant et en riant, ne cherche-t-on pas en effet à atténuer la force polémique de son discours et à rééquilibrer la charge de la modalisation instaurée par des tiers à soi-même (vis-à-vis du fait de croire pouvoir prendre la parole)?

En poursuivant notre étude de cas, la sémiotisation des émotions en interaction pourrait être appréhendée dans une approche spatiale et temporelle, en observant comment se construit et s'explore une antichambre interprétative des affects et des passions [9, p : 343], à savoir un espace où l'on co-construit la valorisation d'un soi sur la crête des interprétations rendues publiques et celles gardées privées; un espace complexe (i) où l'on négocie le regard porté sur soi (espace récursif) et (ii) où l'on partage transitivement les émotions collectives (espace interstitiel). Dans la cogestion des effets de sens de ses propres états comme de ceux des autres, ces frontières entre les positionnements énonciatifs d'un interactant pourraient être appréhendées non pas comme des lignes franches et nettes, mais comme un espace du soi qui gagne ou perd du terrain selon les configurations, comme une topique qui s'épaissit ou se rétrécie en devenant :

- un seuil émotif à préserver, dans une interaction où ne pas brûler des étapes tacitement admises au risque de faire basculer la finesse de l'ironie dans un cynisme brutal ;

- un lieu affectif à expérimenter où entretenir des rapports stables avec les autres acteurs de manière à ce que chacun puisse y trouver une place ;

- un territoire passionnel et identitaire à sillonner et à habiter en tant que tel, où cultiver un sens à partager de manière concertée (mais pas forcément consensuelle).

\subsection{La cogestion des affects : la "bonne distance" et les gestes énonciatifs}

Dans le passage d'un rôle à un autre, les participants repèrent ou imposent des points de transition pertinents en vue d'un échange en public sur des conceptions philosophiques et axiologiques des pratiques urbaines, sur des manières de vivre, de construire et de s'approprier la ville. Par exemple dans l'extrait étudié, la participante, en se créant l'opportunité d'une prise de parole sur les interactions entre aménageurs des espaces 
publics et skateurs, dévie le cours de la balade menée par le duo de skateurs/guides tout en montrant qu'elle en a bien conscience et s'en excuse, d'une certaine manière.

Pour la recherche en sciences du langage sur la sémiotisation des émotions, la médiation culturelle de la balade urbaine guidée affirme son intérêt à la fois sur le plan micro-interactionnel et macro-interactionnel. Sur le plan micro-interactionnel, on s'intéresse aux pressions pragmatiques et affectives qui agissent sur le déroulement chaque balade. Il s'agit alors de caractériser les transformations internes à chaque parcours en notant les points décisifs dans la gestion collective des relations entretenues à l'espace en train d'être traversés (les modalités d'attention et les discours qui portent sur les sites urbains) comme des relations entretenues entre les participants (les formes de socialisation entre les membres du groupes). Sur le plan macro-interactionnel, on se demande notamment si la mise en discours des relations que les habitants et les aménageurs entretiennent au milieu urbain dans le cadre des balades urbaines guidées sur le thème de l'Esprit skate s'approche ou s'éloigne de balades urbaines sur d'autres thématiques; s'approche ou s'éloigne de la mise en discours opérée dans d'autres dispositifs de médiation et de circulation de paroles habitantes. Le terrain d'étude pourrait notamment être élargi et mis en contraste avec le dispositif du « Parlons-en » grenoblois, dont certaines dynamiques de l'imbrication émotions-paroles-actions ont été étudiées dans une

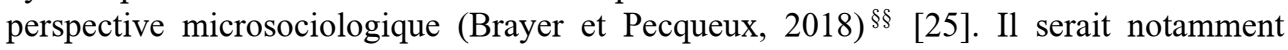
pertinent d'opérer des comparaisons sur le plan de la gestion du groupe (une médiation guidée de l'expérience vs. une animation de débats), du point de vue thématique (une thématique programmée par des guides et un musée vs. des thèmes choisis à l'initiative des personnes présentes), comme du point de vue des interactions entre les participants et leur environnement (des balades urbaines dans la ville vs. des réunions ponctuelles dans une salle).

Une attention portée aux stratégies et tactiques énonciatives mises en œuvre par les participants dans la sémiotisation des émotions permet de caractériser la cogestion des ressources sémiotiques par lesquelles chacun.e négocie la "bonne distance" (Jaillet, 2009; Fontanille, 2014) [26; 27] à l'espace interactionnel, par une prise sur l'environnement au bon moment, entre une fluidification et une coagulation des valeurs promues en discours. En effet, il n'y a pas de posture, d'attitude et, mieux, de modalisation (Goffman, [1974] 1991) [10] qui pourraient être dites appropriées (proper) en elles-mêmes ${ }^{* * *}$. À travers notre recherche dont nous avons présenté seulement ici une partie, nous observons plutôt qu'il y a une posture, une attitude et une modalisation appropriées selon un environnement, qui ont été identifiées et reconnues par tout ou partie des agents qui cohabitent le milieu en train de se former au fil de l'expérience collective.

Aussi, à partir de nos observations, nous nous interrogeons sur la nature des liens entre habitudes et ruses, ou stratégies et tactiques avec Michel de Certeau (1980) [28], en nous

\footnotetext{
$\S \S$ Il est alors important de mentionner ici les motivations selon lesquelles les auteurs d'un chapitre de l'ouvrage sur La démocratie des émotions parlent de « circulation » et non d'une seule circulation des émotions selon un principe « continuiste» :

«D'une part, au Parlons-en, tout ne revient pas à des expressions d'émotions. D'autre part, le principe continuiste « émotions-paroles-actions » vise à ne pas différencier dans nos comptes rendus et analyses ce que les agents ne différencient pas dans le cours des débats; par exemple, à ne pas introduire une gradation ou une temporalité entre ces éléments (du type : d'abord l'émotion, puis la parole, puis éventuellement une action) » [25, p. 76].

*** Erving Goffman s'est intéressé aux dynamiques à travers lesquelles des agents sociaux (re)définissent les cadres de leurs interactions sur les plans matériels, affectifs et symboliques à travers la notion de reframing, en lien étroit avec la notion d'engagement dans l'action :

« Nous avons chacun notre style, ce n'est pas toujours le même et en termes de cadres il s'agit de traductions (rendition), de petites modalisations d'une forme prescrite. Ces changements d'humeur, ces touches personnelles que nous mettons à nos engagements sont généralement autorisés et ont leur importance » [10, p. 125-126].
} 
demandant notamment quels pourraient être les indices linguistiques et sémiotiques à partir desquels repérer une transformation de la ruse en habitude, et vice versa. En effet, tel que l'énoncé «on ne m'écoute pas non je rigole» émerge dans le cours de l'interaction, l'enchaînement entre la culpabilisation des autres et l'excuse est si furtif qu'il semble être une réponse presque automatique à une pression exogène exercée par les autres membres du groupe. Nous nous demandons alors si, dans la transformation et la sémiotisation de ses propres états émotionnels en public, il n'y aurait-il pas une boucle rétroactive des ruses (qui progressent toujours en terrain inconnu) vers les habitudes (qui agissent dans un lieu maîtrisé et intériorisé), finissant ainsi par devenir une parole "entendue", routinisée : si elle est dite et entendue maintes fois sur ce ton, l'expression « non je rigole» perd-t-elle de sa force illocutoire, comme une salutation ou un sourire adressé par convention plus que par intérêt certain? Et quand bien même elle serait automatique, n'est-elle pas pour autant attendue et requise, dans une préservation réciproque des liens sociaux et dans la marque d'un respect mutuel entre les interactants ? Aussi, dans quelle mesure une action qui pourrait être perçue comme ruse et comme geste énonciatif sur une scène spécifique ("on n'a jamais perçu/vécu ça de cette manière") continue-t-elle de l'être pour soi et pour autrui, sur une autre scène?

\section{Ouverture. Les affects et les ruses selon une éthique située}

Finalement, dans l'analyse de l'instauration d'une telle relation interactorielle, on voit qu'il n'est pas très aisé de caractériser les jeux qui se déploient sur plusieurs terrains à la fois : la polémique (feinte ou non) prend appui sur un dialogisme qui, dans un ajustement de la trajectoire d'une action, fait s'entremêler une hétérogénéité de formes sémiotiques. Dans une étude sémiotique qui porte une attention particulière à la dimension énactée des positionnements assumés, revendiqués et imputées en situation, les liens entre émotions et actions mériteraient d'être appréhendé à l'aune de ce que Pierluigi Basso Fossali [29] propose d'appeler «l'agir éthique », une prise sur l'environnement à travers un engagement individuel dans l'espace de l'interaction qui ne saurait totalement se déployer sous les hospices de la morale, ni se plier à des conventions collectives enracinées - en ce que cet agir lui-même est soumis à une (re)définition de sa propre force et de sa propre valeur in situ. Celui-ci n'est alors jamais indissociable des «émotions éthiques » qui concernent la définition démocratique d'un «co-sens» de ce qui est en train d'être éprouvé en interaction, l'élaboration collective d'un « horizon du juste », à savoir un destin justement partageable parce qu'il respecte le caractère spécifique de chaque trajectoire biographique sur le plan individuel. Ainsi, la sémiotisation de ses propres émotions est toujours en prise avec celle des émotions d'autrui/par autrui, raison même pour laquelle il y a toujours une indétermination à pouvoir programmer en amont et anticiper de manière assurée le parcours de signification de la sémiose opérée en groupe ${ }^{\dagger \dagger}$.

Dans l'entremêlement, d'un côté, entre la diversité des rôles potentiels que l'on peut soi-même jouer in vivo et, de l'autre côté, entre la diversité des rôles que les autres peuvent tenir, le caractère approprié d'une conduite en interaction, au regard des émotions qu'elle suscite, ne sera jamais totalement prévisible. Quand bien même on cherche à les canaliser, voire à les gouverner, les émotions ressortissent d'une tension constante entre ce qui est

\footnotetext{
${ }^{\dagger \dagger \dagger}$ On peut citer ici le passage auquel nous faisons référence, de manière à ce que le lecteur puisse saisir la mise en réseau du concept d'éthique avec celui de forme de vie :

" Aux émotions qui concernent la mise en phase d'une plate-forme éthique des traductions entre la destinalité de diverses formes de vie, on peut ajouter les émotions enracinées dans la « compréhension » de la trajectoire comportementale d'autrui, étant donné que la mise en jeu de ses propres soi-ipse possibles comme moyen de traduction de l'altérité finit par les racheter, les affranchir d'une archéologie existentielle douloureuse, voire coupable ». (Basso Fossali, 2008 : 64) [29]
} 
déjà sémantisé, à partir ce qui est vécu ou à vivre (un plan de l'existence) et ce qui est en train d'être sémantisé, à partir de ce qui est en train d'être vécu ou rééprouvé (un plan de l'expérience), tension à laquelle on ne peut (r)échapper - sur son versant dysphorique - ou dont on ne saurait se priver de jouissance - sur son versant euphorique. Dans une perspective phénoménologique de la sémiose des émotions, les passages entre les parts publiques et privées d'une vie en société impliquent une articulation entre une observation de premier ordre (d'où émergent les émotions, vécues, en étant plongé dans l'interaction) et une observation de deuxième ordre (d'où émergent les affects, en portant une attention à rebours sur la scène).

En écho à la réflexion métalinguistique opérée en ouverture de cet article, il est ici opportun, voire nécessaire, de poursuivre notre réflexion épistémologique sur la sémiose des émotions appréhendée, d'une part, en tant que procès interprétatif et, d'autre part, en tant qu'opération analytique. $\mathrm{Si}$, sur le plan individuel, « le procès ouvert de l'interprétation cherche à être "urbanisé" à travers la prophylaxie de l'analyse » [30, p. 129], il ne peut y parvenir pour autant sur le plan de la collectivité, en ce que le caractère diffractant de la multiplicité des points de vue semble être déjà perçu et intériorisé comme tel par les agents qui prennent part à la sémiotisation d'une interaction. En effet, par le recours à des formes comme l'ironie ou l'autodérision qui progressent sur au moins deux plans de signification, les pratiques des agents sociaux font montre des tensions (i) entre leurs propres expériences socioaffectives et sociolangagières de l'espace interactionnel et celles des autres (perception en interaction); (ii) entre leur propre interprétation de la scène et celles des autres (interprétation et énonciation); (ii) entre le discours réflexif qu'ils produisent sur leur propre positionnement actoriel et les discours que les coparticipants pourraient élaborer (élaboration collective d'un récit sur la ville, d'un récit dans la ville et gestion publique des ethos).

Dans une approche transversale de la sémiotisation des émotions en sciences du langage, au-delà du commentaire sur son propre regard de chercheur, une réflexion reste encore à mener vis-à-vis de la focalisation opérée sur les faisceaux d'indices sur lesquels s'appuient les acteurs sociaux eux-mêmes pour négocier le sens de leurs propres expériences, sur les mimiques, les orientations corporelles, les mouvements du corps et les silences à partir desquels ils infèrent des formes d'engagements dans la situation et des positionnements éthiques. Une telle réflexion permettrait alors d'interroger (i) la tenue des liens sociaux cogérés in vivo avec les acteurs de l'enquête, à savoir les modalités par lesquelles les chercheurs tissent des liens socio-affectifs avec les participants dans le cours de l'interaction; (ii) les conditions environnementales du déploiement de la recherche, à savoir la manière dont les soubassements sociohistoriques d'une épistémologie se trouvent transformés par un regard transitif ou réflexif sur les théories et méthodes mises en œuvre ; (iii) et le devenir de ses propres analyses pour le compte de la collectivité, à travers une réappropriation publique et critique des descriptions et des modélisations opérées.

\section{Bibliographie}

1. C. Kerbrat-Orecchioni, Les interactions verbales (Armand Colin, Paris, 1992).

2. E. Goffman, La mise en scène de la vie quotidienne (Minuit, Paris, 1973).

3. M. Detienne et J.-P Vernant. Les ruses de l'intelligence. La mètis des Grecs, (Flammarion, Paris, 2009).

4. A. J. Greimas et J. Fontanille. Sémiotique des passions. Des états de choses aux états d'âme, (Seuil, Paris, 1991)

5. T. Ingold, Marcher avec les dragons (Zones Sensibles, Bruxelles, 2013).

6. A. Pecqueux, Tordre l'attention. Ajustements perceptifs en situation. Dans Y. Citton (dir.), L'économie de l'attention. Nouvel horizon du capitalisme?, 219-228 (La Découverte, Paris, 2014) 
7. R. Micheli Les émotions dans les discours. Modèle d'analyse, perspectives empiriques, (De Boeck, Bruxelles, 2014)

8. P. Basso Fossali et O. Le Guern (éds), L'appropriation sémiotique. L'interprétation de l'altérité et l'inscription du soi (Lambert-Lucas, Limoges, 2018)

9. J. Thiburce, De l'usage tacite des espaces publics aux formes d'appropriation narrative et affective de la ville, Thèse de doctorat de sciences du langage sous la dir. de P. Basso Fossali, Université Lumière Lyon 2, UMR 5191 ICAR, ED 484 3LA. (2018)

10. Goffman E, Les Cadres de l'expérience, (Minuit, Paris, 1991)

11. H. Garfinkel, Studies on ethnomethodology (Polity Press, Cambridge, 1984)

12. L. Mondada, L'énonciation comme phénomène émergent dans l'interaction : le cas des pre-beginnings. Dans M. Colas-Blaise, L. Perrin, G.-M. Tore (dirs.), L'énonciation aujourd'hui. Un concept clé des sciences du langage, 317-340 (Lambert-Lucas, Limoges, 2016)

13. J. Heritage, A change-of-state token and aspects of its sequential placement. Dans J. Maxwell Atkinson et J. Heritage (eds) Structures of Social Action: Studies in Conversation Analysis, 299-345 (Cambridge University Press, Cambridge, 1984)

14. F. Rastier, Formes sémantiques et textualité. Langages, 163, 3, 99-114 (2006).

15. A. Lescano, Pour une étude du ton. Langue Française, 164, 4, 45-60 (2009).

16. J. Longhi, Monde, discours, "sujet énonçant" : quelles modalités de constitution pour la conscience ?. Dans P. Frath, J. Pauchard et L. Lansari, Res Per Nomen II, 251-264 (Éditions des Presses Universitaires de Reims, Reims, 2011)

17. E. Benveniste, Problèmes de linguistique générale 1 (Gallimard, Paris, 1966)

18. A. Berrendonner, Éléments de linguistique pragmatique (Minuit, Paris, 1981)

19. O. Ducrot, Le Dire et le Dit, (Minuit, Paris, 1984)

20. D. Maingueneau, L'ethos discursif et le défi du Web. Itinéraires [En ligne], 3. (2016) URL : http://journals.openedition.org/itineraires/3000

21. R. Amossy, La Présentation de soi. Ethos et identité verbale (PUF, Paris, 2011)

22. J. Bres, Dialogisme. Dans C. Détrie, P. Siblot et B. Verine (éds), Termes et concepts pour l'analyse du discours, 83-86 (Champion, Paris, 2001)

23. P. Basso Fossali, Vers une écologie sémiotique de la culture. Perception, gestion et réappropriation du sens (Lambert-Lucas, Limoges, 2017)

24. P. Basso Fossali, Le geste et sa niche : gestion du sens « hors technique ». Texto! Textes et Cultures, Équipe Sémantique des textes, Volume coordonné par C. BaltaretuThénault et C. Cusimano, vol. XXII, n². (2017)

25. L. Brayer et A. Pecqueux, Le Parlons-en comme espace de circulation. Dans Loïc Blondiaux (éd.), La démocratie des émotions: Dispositifs participatifs et gouvernabilité des affects, 67-91 (Presses de Sciences Po, Paris, 2018)

26. M.-C. Jaillet, Contre le territoire, la "bonne distance". Dans M. Vanier (dir.), Territoires, Territorialité, Territorialisation. Controverses et perspectives, 115-121 (PUR, Rennes, 2009)

27. J. Fontanille, Territoire. Dans E. Mitropoulou et E. Landowski (dirs.), Actes sémiotiques, $\mathrm{n}^{\circ} 117,(2014)$.

28. M. Certeau, L'invention du quotidien 1 : arts de faire (Gallimard, Paris, 1980)

29. P. Basso Fossali, Éthique et sémiotique des destins croisés. La négociation de l'agir sensé entre formes de vie. Protée. Revue internationale de théories et de pratiques sémiotiques, 36, $\mathrm{n}^{\circ} 1$, Éthique et sémiotique du sujet, 59-69 (2008)

30. P. Basso Fossali. L'interprétation dans son espace phénoménologique : jeux de langage et implémentation publique. Metodo, 3, n. 1, "Phenomenology and semiotics", 113138 (2015) 